\title{
The Neurological Aspects of COVID-19: Do Women Respond Differently?
}

\author{
Aastha Takkar ${ }^{1}$ Monika Singla² \\ ${ }^{1}$ Department of Neurology, Post Graduate Institute of Medical \\ Education and Research, Chandigarh, India \\ ${ }^{2}$ Department of Neurology, Dayanand Medical College and \\ Hospital, Ludhiana, Punjab, India
}

\begin{abstract}
Address for correspondence Aastha Takkar, MD, DM, Department of Neurology, Post Graduate Institute of Medical Education and Research, Chandigarh, 160012, India (e-mail: draastha49@yahoo.com).
\end{abstract}

Ind J Car Dis Wom:2020;5:264-270

\begin{abstract}
Neurovirulence of Coronavirus-2(SARS-CoV-2) was established soon after the Coronavirus disease-19 (COVID-19) pandemic broke. While the initial reports from Chinese cohorts suggested that around 8 to $36 \%$ patients of COVID-19 develop neurological complications, upcoming literature revealed the various neurological presentations this newly emergent virus could have. Data on neurological manifestations of COVID-19 is still

Keywords

- COVID-19

- neurological manifestations

- SARS-CoV-2

- stroke

- women accumulating. Despite an immense flooding of recent databases, gender-specific issues of this novel virus still remain elusive. Increased mortality has been noted in elderly male patients, and immediate risk of death is a major concern. As per the available literature, women are more likely to recover from the virus, but little is described about the medical and social repercussions. Gender-based analysis should help delineate specific problems in women in the rapidly changing scenario. This article discusses the relevant neurological aspects of COVID-19 with specific reference to women.
\end{abstract}

\section{Introduction}

Neuroinvasiveness of Orthocoronavirinae subfamily, severe acute respiratory syndrome Coronavirus- 1 (SARS-CoV-1) and Middle East respiratory Coronavirus (MERS-CoV), has been well-established. When the severe acute respiratory syndrome corona virus-2 (SARS-CoV-2)/Coronavirus disease-19 (COVID-19) pandemic broke, neurological repercussions were expected round the corner. ${ }^{1}$

Initial reports from Chinese cohorts suggested that around 8 to 36\% patients of COVID-19 develop neurological complications. With upcoming literature on varied presentations of this newly emergent virus, we are getting wiser about the myriad manifestations it can have. ${ }^{2}$ Despite an immense flooding of recent databases, gender-specific issues of this novel virus still remain elusive. Gendered effects of a pandemic are well-expected at all fronts-social, physical, financial and, of course, medical.

\section{Search Strategy and Methodology}

Medical databases were searched for relevant literature using the following key words: "Coronavirus," "COVID-19,"
"Neurological," "Gender," “Women," “Encephalopathy," and "CNS." Original studies, case reports, review articles, letters to editor, and neurological images were selected for review. This manuscript presents a narrative review along with a neurologist's perspective on COVID-19 in women.

\section{Neurological Manifestations of COVID-19}

What started as an unexplained pneumonitis in territories of Wuhan, rapidly spread beyond the geographical boundaries and was declared as a pandemic in March 2020. By the time the COVID-19 pandemic was inevitable, literature had already accumulated, suggesting the wide spectrum of manifestations COVID-19 can have. Extrapolation of studies on other viruses in the orthocronaviradae group like SARSCoV-1 and MERS also suggested the neurotropic potential of the novel SARS-CoV-2.

Neurovirulence of COVID-19- the Etiopathogenesis Neurological manifestations of COVID-19 are unique in exhibiting additional pathophysiological aspects. While the exact pathogenesis is still not established fully, the primary target of COVID-19 is believed to be the respiratory epithelium.

(C)2020 Women in Cardiology and Related Sciences
License terms

(ㅇ)( $\Theta \circledast$ 
Neurological complications are considered secondary and may arise due to direct invasion, immune-mediated damage or secondary complications like hypoxia or shock.

\section{a. Direct Neuroinvasion}

Neurotropism of SARS-CoV-2 has been well-established. It should be remembered that the brain becomes more susceptible to direct invasion when the blood-brain barrier is disrupted due to an uncontrolled systemic inflammatory response. The routes by which it enters the central nervous system (CNS) are as follows: ${ }^{3,4}$

- Olfactory epithelium: Retrograde entry to CNS may occur through cribriform plate

- Cellular infection and invasions: The infected macrophages act as a viral reservoir and spread to the brain. Both hematogenous as well as lymphatic routes have been described

- Endothelial cells of the blood-brain barrier are known to express SARS-CoV receptors-the angiotensinconverting enzyme-2(ACE-2) and CD209L. ACE-2 is also expressed in glial cells, cerebral blood vessels, spinal cord, and skeletal muscles. ${ }^{5}$

- Transsynaptic route: Transmission may also occur through the peripheral nerves

b. Immune-mediated Injury-An aberrant immune-mediated response may be incited, giving rise to many parainfectious/postinfectious neurological complications.

The virus effectively evades the host's immune response, while the uncontrolled systemic inflammatory response/cytokine storm is a major cause of mortality in COVID-19. ${ }^{6}$

c. Systemic Involvement: Severe respiratory distress causes systemic hypoxia. Hypercarbia and vasodilatation are indirect consequences, worsening the anerobic metabolism and causing neuronal cell death. SARS-Cov-2 can lead to multiorgan failure and systemic involvement, causing uremic encephalopathy or hypoxic encephalopathy. Disseminated intravascular coagulopathy (DIC)-mediated intracranial bleed or thrombosis is another major cause of mortality in these patients.

\section{Women-Related Issues}

\section{Pathophysiology: Does COVID-19 Affect Women Differently?}

Despite the recent flooding of literature on COVID-19, women-specific epidemiological issues and prevalence patterns have not been discussed. Min Jin et al in their review highlighted that while men and women have similar prevalence, men with COVID-19 are at a 2.4 times higher risk for worse outcome and death, independent of their age. ${ }^{7,8}$ Similar findings have been reported from Italian and American cohorts

While some have considered this as a general demographic fact, others have implicated higher circulating levels of ACE-2 in men.
Li et al compared ACE-2 expression levels across 31 normal human tissues and concluded that there was no significant difference between male or female genders across all ages, indicating that, per say, the infection risk of SARS-CoV-2 may not have any significant association with gender. The discrepancy in mortality rate has been considered due to differences in adaptive and innate immune responses. ${ }^{8,9}$

Also, of relevance is the fact that the LGBTQI community is at a higher risk due to these issues and a higher prevalence of premorbid diseases like cancer and HIV and reluctance to seek medical care may make them further prone to a more severe illness.

\section{Neurological Manifestations of COVID-19- the Neurological Syndrome}

The list of neurological manifestations in COVID-19 has been increasing over the past few months. Varying incidences of neurological manifestations have been observed in the available literature. In one of the initial retrospective series, Mao et al reported neurological complications in $36.4 \%$ of patients. ${ }^{2}$ Helms et al in their observational study reported a much higher incidence (84\%) of neurological involvement. ${ }^{10}$ Many neurological complications (other than metabolic or hypoxic encephalopathy) tend to occur early in the disease course and in general, neurological manifestations have been associated with a severe respiratory infection. Practically, every part of both central and peripheral nervous system (CNS and PNS) can be involved in COVID-19. - Fig. 1 shows an overview of potential neurological complications in a patient of COVID-19.

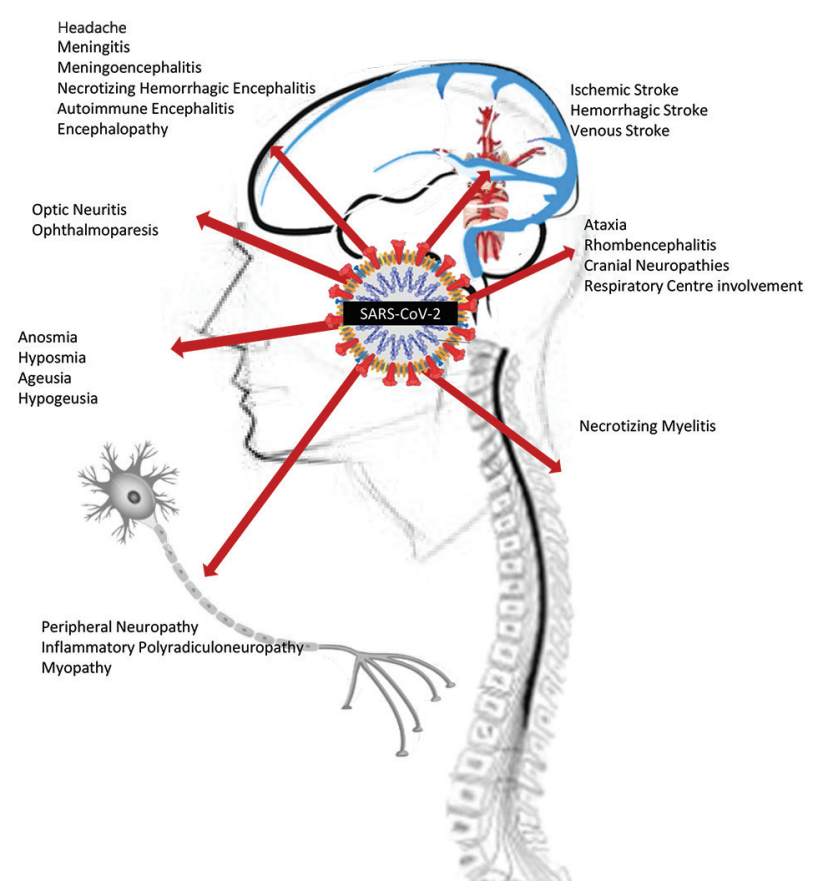

Fig. 1 An overview of neurological manifestations of COVID-19 


\section{CNS Manifestations}

CNS manifestations may arise due to focal or diffuse involvement of meninges, parenchymal structures, spinal cord and involvement of cerebral vasculature. CNS involvement is rather the most common neurological presentation. Symptomology may vary, depending upon the anatomical substrate involvement.

Headache and dizziness are the most commonly described symptoms of neurological involvement. Incidence of headache among various available reports ranges from 8 to $13.6 \%$.,11 Meningeal irritation, meningeal inflammation, and increased intracranial pressure have been postulated as possible mechanisms causing headache. Worsening of an underlying primary headache syndrome, new onset personnel protective equipment (PPE)-related headache, stress, and tension type headaches can be other causes of headache. A thorough clinical history and examination should be done in all patients for an early recognition of the ominous causes of headache.

Meningeal involvement causing meningitis and meningoencephalitis have also been reported in observational studies, case series and case reports. ${ }^{10}$ Seizure and altered sensorium are common presentations. SARS-CoV-2 RNA was detected in cerebrospinal fluid (CSF) of a few patients of COVID-19 mediated meningoencephalitis. Viral clearance in CSF may precede the neurological involvement and therefore the detection may be delayed. ${ }^{12,13}$

Acute necrotizing hemorrhagic encephalitis related to intracranial cytokine storm may occur and has been related to a worse prognosis..$^{14}$ Dogan et al also considered autoimmune encephalitis in their six patients, based upon their clinical symptomatology and response to plasmapheresis. ${ }^{15}$ Another case of anti-NMDA-R antibody positivity was reported by Panareillo et al. These findings highlight the importance of activation of an indirect pathway involving the immune system in SARS-CoV-2 infection. ${ }^{16}$

Rhombencephalitis causing ataxia, leukoencephalopathy with diffuse corticospinal involvement, delirium, executive disturbances, behavioral disturbances, sleep disorders, dementia, and movement disorders are other syndromes associated with COVID-19. ${ }^{17}$

No conclusive laboratory (including CSF analysis) or neuroradiological investigations have been described. In a recently published series by Franke et al, varied neurological symptoms like myoclonus, oculomotor disturbances, delirium, dystonia, stroke, seizures, etc., were noted in 11 severely ill COVID-19 patients. They also noted increased levels of neurofilament light chain in the CSF of these patients. All patients in their cohort had antineuronal autoantibodies in serum or CSF. ${ }^{18}$

In a multi-institutional French cohort, authors reported various radiological abnormalities, including medial temporal lobe hyperintensities, nonconfluent multifocal white matter hyperintense signal intensities, and extensive, isolated white matter hemorrhages in nonstroke patients of COVID-19. ${ }^{19}$

\section{Vascular Involvement}

Of particular interest is the cerebrovascular involvement with COVID-19. Incidence of strokes reported in European and Chinese hospital COVID-19 registries is up to 2.5 to $6 \%$. Mao et al in his cohort noted strokes in 6 (2.8\%) out of 214 hospitalized COVID-19 patients (5 ischemic and 1 hemorrhagic stroke). These were large vessel strokes and were seen in association with severe COVID-19 illness. Cortical venous thrombosis has also been reported in small series. ${ }^{20}$ In the series by Oxley et al, large vessel strokes were noted in four men and one woman. In their cohort, all reported strokes were in the middle cerebral artery territory. No typical symptoms of COVID-19 infection were present at the outset in 3 of the 5 patients. $D$ dimer was raised in all but one.

The proinflammatory and prothrombotic mechanisms causing stroke in COVID-19 have aroused a great deal of interest. Endothelial dysfunction, DIC and thrombotic microangiopathy associated with thrombocytopenia, elevated D-dimer and prolonged prothrombin time have been hypothesized as precursors. With profoundly elevated D-dimer levels, COVID19 has been considered as a highly prothrombotic state. Combined with factors like immobilization, hypoxia, inflammation and stress, accelerated thrombosis has been seen to occur in severely ill/ICU-bound patients. Role of antiphospholipid antibodies causing multifocal infarction has also been suggested by few. Prevalence of lupus anticoagulant in as high as $91 \%$ patients has been reported. Autopsy studies have suggested associated venous thrombosis in lower limbs, lungs and pulmonary circulation. Endothelitis in kidney and small intestine vessels has also been reported. Extrapolating these findings to cerebral vasculature may explain the etiopathogenesis of ischemic and hemorrhagic arterial and venous strokes. ${ }^{21-23}$

\section{Women-Relatedlssues}

\section{Stroke in COVID-19- ARE Women Different?}

Pathophysiology of stroke has been gendered for a long time. The risk of cardioembolic strokes in women is higher as compared with men but they are comparatively protected for noncardioembolic strokes, due to the circulating estrogen. Also, due to differences in consumption of both alcohol and tobacco (lower in women) and lower rates of both dyslipidemia and diabetes in women, they are guarded against developing ischemic strokes till around 75 years. Another significant cause of stroke in young women in reproductive age group is a venous stroke (cerebral venous thrombosis).

From our perspective, the COVID-19 pandemic has probably caused reshifting of gender-epidemiology of stroke. The available data has provided another major variable in stroke pathogenesis-the inflammatory cascade in the background of COVID-19 pandemic, which probably has an equal predisposition for men and women. The activation of inflammatory and coagulation cascade probably depends upon the severity of infection. The pre-existing stroke risks might be causing additive effects on causation but no discrete data are available to its say. 
Brainstem and Spinal CORD Involvement

A handful of case reports of patients with necrotizing myelitis are available. It has been postulated that the severe and refractory gastrointestinal or respiratory involvement could be contributed by affected central pathways involving area postrema or chemoreceptor trigger zone. ${ }^{24}$ Mortality associated with respiratory failure has been postulated to be neurogenic, resulting from involvement of respiratory centers of the brain.

\section{Peripheral Nervous System}

COVID-19 may cause isolated cranial neuropathies; specifically, olfactory nerve involvement has received special focus. Concerns of spread of infection during endonasal procedures have been raised since the early days of suspected community transmission. ${ }^{25}$ Olfactory and taste dysfunction are considered as early symptoms of COVID-19 and studies have reported variable involvement (5-88\%). Anosmia, hyposmia, parosmia and phantosmia may occur (in descending order). Apart from suspected immune, local inflammatory and direct cytopathic responses, altered neurotransmission affecting serotonin/dopamine pathways and altered central pathways of olfaction have been implicated. Other cranial neuropathies causing optic or ocular nerve involvement have been infrequently reported.

Acute inflammatory demyelinating or axonal radiculoneuropathy/Guillain-Barre syndrome and its variants (Miller-Fisher Syndrome) have been noted, probably due to an aberrant immune response.

A large proportion of patients have reported myalgia and fatigue (14.9-44\%) in various case series and observational studies. Myopathy was diagnosed on the basis of raised CPK (> $200 \mathrm{U} / \mathrm{L}$ ) in $10.7 \%$ of Chinese patients who presented with weakness and fatigue. ${ }^{2}$ Muscle involvement can be because of direct invasion or as a result of extensive inflammation.

\section{Neurological Complications Due to Secondary/ Systemic Involvement}

Hypoxia, hypotension or multiorgan failure related encephalopathy needs due consideration as it is a major cause of mortality. Uremia and acute respiratory distress syndrome (ARDS) may themselves be associated with many metabolic alterations. Similarly, critical illness associated neuropathy and myopathy also deserve mention, keeping in mind prolonged ventilatory support, immobilization and nutrition deprivation in these patients.

\section{Depression and COVID-19}

COVID-19 has negatively affected society's mental health to a large extent. Uncertain disease course and prognosis, persistent shortage of resources for diagnosis and personnel protective gears, and limited available treatment options have been disturbing both for the care providers as well as patients. Mass home confinement, excessive media exposure, limited access to public/social gatherings and resultant financial losses during the pandemic have negatively contributed toward mental health. In a cross-sectional study involving 81 participants, depression was noted in $23.6 \%$ patients and $45.1 \%$ patients reported anxiety. Similar findings were noted in other studies. ${ }^{26}$ Healthcare workers are in particular at higher risk. In a multinational, multicenter study of 906 healthcare workers, Chew et al noted an extremely high-incidence of stress, anxiety, headache and lethargy. ${ }^{27}$

\section{Women-Related Issues}

\section{Course of the Disease: Do Women Respond Differently to COVID-19?}

As discussed, the virus invades both genders in probably a similar way. No specific gender differences have been noted in most of the CNS and PNS manifestations of COVID-19 although the complications due to the immune-mediated responses may differ in men and women. Men are more predisposed to complications. Sex hormones may modulate the expression of ACE2 , as suggested by La Vignera et al. ${ }^{28}$ Role for androgen receptor gene polymorphisms need to be excluded in the pathogenesis of SARS-CoV-2. ${ }^{29}$

The immunological aspect of sex hormones has also been under scrutiny in COVID-19 times. It has been strongly suggested that immune regulatory genes, coded by $\mathrm{X}$ chromosomes, result in a generally strong immune response in women. The complex immune- modulating role of estrogen may be causative in exhibiting an antiinflammatory response. ${ }^{30}$

In believing the protective role of estrogens, another caveat in the epidemiological observations is the probable protective impact on young male patients. It is possible that akin to the estrogen effects in young female patients, testosterone also has protective anti-inflammatory effect in younger men. Analogous to estrogen, testosterone has been noted to decrease IL-6 and TNF-a via inhibition of the NF-kB pro-inflammatory pathway. ${ }^{31}$

Gender-based lifestyle choices, differences in environmental factors like smoking, more social mobility, risk taking behavior, and nonfollowing strict public health measures make men more susceptible to infection. These are some additional issues which have been raised in literature.

Women have been noted to be more prone to depression and anxiety. Excessive societal demands in the prevailing pandemic has probably caused further worsening. A cross-sectional study evaluated the prevalence of depression, anxiety and health anxiety in Turkish society and concluded that women are more affected psychologically. Prioritizing mental health in the high-risk group is required. ${ }^{26}$

\section{COVID-19-the Impact on Underlying Neurological Illnesses}

Patients with long-standing neurological illnesses like motor neuron disease, myasthenia gravis, Parkinson's disease, dementia, inflammatory neuropathy, and myopathy may have reduced mobility, disturbed swallowing and 
compromised respiratory functions. COVID-19 infection may cause further worsening of the underlying compromised lung functions.

Immunosuppressive modalities have been widely used in contemporary neurology. Immune dysregulation is seen in neuroimmunological disorders like multiple sclerosis (MS), neuromyelitis-optica spectrum disorder, myasthenia gravis, autoimmune encephalitis, inflammatory neuropathies, and myopathies. In addition, these patients are often on chronic immunosuppressive therapies. This cohort is not just prone to get superadded infections, but once infected may also exhibit a poorer prognosis. Interestingly, some disease modifying therapies causing moderate immunosuppression, used in MS, may provide a beneficial effect in preventing complications related to exuberant immune response, considering potential antiviral properties trials of Interferon $\beta$ are underway. Fingolimod is also been considered as treatment for ARDS related to COVID-19. ${ }^{32}$ An immune-mediated illness may respond erratically to physical and mental stress, worsening its own disease course and causing significant morbidity.

In a community-based case control study on COVID-19 patients, it was concluded that patients with an underlying Parkinson's disease experience substantial worsening of their motor and nonmotor symptoms. Altered pharmacokinetics of the dopaminergic treatment or infection-related systemic inflammatory response were postulated as probable mechanisms. ${ }^{33}$

Stress-related worsening of neurological diseases, particularly Parkinson's disease, dementia, MS and epilepsy, is a well-established fact and needs to be considered while attending to these patients. ${ }^{34}$

\section{Women-Related Issues}

\section{Chronic Dementia and COVID-19: Do Women Suffer Differently?}

It is well-known that both estrogen and testosterone levels relate with cognitive performance. Hormones may enhance, modulate, and depress specific cognitive skills. Data with regard to the COVID-19 pandemic and differential cognitive decline is not available; in general, men with dementia have been found to outperform women counterparts in several cognitive domains like language, visuospatial abilities and episodic memory. The reduced estrogen after menopause, greater cognitive reserve in men, and role of apolipoprotein $\mathrm{E} \varepsilon 4$ allele have been postulated.

\section{Treatment Protocols of Neurological Illness During COVID-19 Pandemic}

The ongoing COVID-19 pandemic has posed major health challenges for all faculties. Reorganization of existing healthcare paradigms and treatment protocols are needed, keeping in mind the safety strategies for healthcare workers (HCWs). Considering the probable neurological emergencies, overwhelmed healthcare facilities, shortage of screening and protective equipment, consensus statements for many neurological illnesses have been proposed by different national and international neurological societies.

Cerebrovascular accidents are a major neurological emergency. The concept of "protected Code Stroke" has taken into account various issues like robust screening, time-sensitive care at primary facilities, telemedicine, designating separate CT machines, intravenous thrombolysis, mechanical thrombectomy guidelines, and telerehabilitation facilities. ${ }^{35}$

Similarly, consensus statement on immunological diseases like MS has focused upon possible risks worsening the immunological disease process, risk of acquiring secondary infections, risks posed due to various immunomodulatory drugs, and risk of relapse. ${ }^{36}$

\section{Neurological Complications Associated with Treatment of COVID-19}

Chloroquine and hydroxychloroquine have been hyped with respect to prophylaxis and treatment of mild-to-moderate disease. The potential spectrum of neuropsychiatric manifestations includes agitation, sleep disturbances, mania, hallucinations, paranoia, depression, catatonia, psychosis, and suicidal ideations. De-novo headaches may occur in nearly $2.8 \%$ of patients. Other minor side effects are dizziness, visual field defects, paresthesia, and insomnia. Toxicity and overdose may cause seizures, syncope, hypokalemia, and cardiovascular collapse. ${ }^{37}$

\section{COVID-19- the Impact on Health Care: Social and Economic Aspects}

\section{Neurological Implications of the Personnel Protective Equipment (PPE)}

In a cross-sectional study involving 158 frontline HCWs, it was noted that PPE was associated with de-novo headache and also exacerbates preexisting headache disorders. ${ }^{38}$ Psychopathological implications of facemask was studied in another nationwide online survey which suggested that use of facemask could provide a sense of false security due to an increased level of self-perceived protection notion. This may adversely undermine other infection control measures $^{39}$

\section{Women-RelatedIssues \\ PPE-Do Women have Similar Neuropsychiatric Implications?}

There is growing concern on the "default-sized unisex PPE" that usually do not fit women. A woman front-liner is more exposed to the risks associated with a faulty PPE that does not suit her built. Ill-fitting PPE (including ill-fitting face masks) also cause excess headache, anxiety, and fear of contracting the illness.

Additionally, no specific concerns have been drawn over a woman's menstrual health while drawing guidelines of PPE usage. These all may be an additional cause of stress and anxiety among women HCWs. 


\section{Neurologist's Perspective of Lockdown in COVID-19 Crisis}

A general view of the lockdown-related issues can be extrapolated from a report published by the neurological alliance of Ireland which discussed the neurological impacts of restrictions imposed during the COVID-19 pandemic. Due to restrictions of physical visit to treating clinicians, limited access for healthcare providers, and withdrawal of home care by providers, $26 \%$ patients had difficulty in accessing neurological care and had reported subjective worsening.

\section{Women-Related Issues}

\section{Public Health Measures and Women}

An overwhelming $70 \%$ of the health and social sector workforce is composed of women. Despite being heavily engaged in medical and social service, major differences in work settings exist. Gender gap in pay are pre-existent and women often form an unpaid (at times unacknowledged) workforce, being forced to work in the home setting and being subjected to gender and domestic violence. Public measures like lockdown may enforce an extra burden caused by school closures, sick members at home, and increased domestic chores, causing increased psychological impact on society, especially women who are major work forces in these scenarios. This may have significant impact on the social, physical and mental health of women.

\section{Conclusions: The Future Perspective}

Data on neurological manifestations in COVID-19 is still accumulating. The course of the disease is unfolding with every passing day, but there is evidence of increased mortality among elderly male patients. Gender specific response to illness looks to be a major factor in determining the differences in morbidity and mortality. For elderly men in general, the immediate risk of death from the disease is the greatest concern. For women, who are more likely to recover from the virus, other repercussions of COVID-19 may unfold subsequently and last for years to come. A consistent analysis of gender-specific data will ensure that specific needs of women, girls and marginalized groups are rendered visible. Woman-specific considerations should be given, while keeping in mind diagnostic, therapeutic and prophylactic measures.

\section{Financial Disclosures/Funding Source \\ None.}

\section{Conflicts of interest}

None declared.

\section{References}

1 Desforges M, Le Coupanec A, Stodola JK, Meessen-Pinard M, Talbot PJ. Human coronaviruses: viral and cellular factors involved in neuroinvasiveness and neuropathogenesis. Virus Res 2014;194:145-158
2 Mao L, Jin H, Wang M, et al. Neurologic manifestations of hospitalized patients with Coronavirus disease 2019 in Wuhan, China. JAMA Neurol 2020;77(6):1-9

3 Sepehrinezhad A, Shahbazi A, Negah SS. COVID-19 virus may have neuroinvasive potential and cause neurological complications: a perspective review. J Neurovirol 2020;26(3):324-329

4 Niu J, Shen L, Huang B, et al. Non-invasive bioluminescence imaging of $\mathrm{HCoV}-\mathrm{OC} 43$ infection and therapy in the central nervous system of live mice. Antiviral Res 2020;173:104646

5 Lahiri D, Ardila A. COVID-19 pandemic: A neurological perspective. Cureus 2020;12(4):e7889

6 Li X, Geng M, Peng Y, Meng L, Lu S. Molecular immune pathogenesis and diagnosis of COVID-19. J Pharm Anal 2020;10(2):102-108

7 Jin J-M, Bai P, He W, et al. Gender differences in patients With COVID-19: focus on severity and mortality. Front Public Health 2020;8:152

8 Chen T, Wu D, Chen H, et al. Clinical characteristics of 113 deceased patients with coronavirus disease 2019: retrospective study. BMJ 2020;368:m1091

9 Chen N, Zhou M, Dong X, et al. Epidemiological and clinical characteristics of 99 cases of 2019 novel coronavirus pneumonia in Wuhan, China: a descriptive study. Lancet 2020;395(10223):507-513

10 Helms J, Kremer S, Merdji H, et al. Neurologic features in severe SARS-CoV-2 infection. N Engl J Med 2020;382(23):2268-2270

11 Guan W-J, Ni Z-Y, Hu Y, et al; China Medical Treatment Expert Group for Covid-19. Clinical characteristics of Coronavirus disease 2019 in China. N Engl J Med 2020;382(18):1708-1720

12 Moriguchi T, Harii N, Goto J, et al. A first case of meningitis/ encephalitis associated with SARS-Coronavirus-2. Int J Infect Dis 2020;94:55-58

13 Espíndola OM, Siqueira M, Soares CN, et al. Patients with COVID-19 and neurological manifestations show undetectable SARS-CoV-2 RNA levels in the cerebrospinal fluid. Int J Infect Dis 2020;96:567-569

14 Poyiadji N, Shahin G, Noujaim D, Stone M, Patel S, Griffith B. COVID-19-associated acute hemorrhagic necrotizing encephalopathy: CT and MRI features. Radiology 2020 (e-pub ahead of print). doi: https://doi.org/10.1148/radiol.2020201187 :

15 Dogan L, Kaya D, Sarikaya T, et al. Plasmapheresis treatment in COVID-19-related autoimmune meningoencephalitis: case series. Brain Behav Immun 2020;87:155-158

16 Anti-NMDA receptor encephalitis in a psychiatric Covid19 patient_ A case report. Available at: https://reader. elsevier.com/reader/sd/pii/S0889159120308734? token=BB 64C 442 B 7FD 351 C5A45BAD 49965C59F8970FBBD 480497CE051D8E2480BFF97D2620B429D7BC2470756A50606D715A5C. Accessed July 31, 2020

17 Bernard-Valnet R, Pizzarotti B, Anichini A, et al. Two patients with acute meningoencephalitis concomitant with SARSCoV-2 infection. Eur J Neurol 2020 (e-pub ahead of print). Doi: https://doi.org/10.1101/2020.04.17.20060251

18 High frequency of cerebrospinal fluid autoantibodies in COVID-19 patients with neurological symptoms. Available at: https://www.medrxiv.org/content/10.1101/2020.07.01.20143 214v1. Accessed July 31, 2020

19 Kremer S, Lersy F, de Sèze J, et al. Brain MRI Findings in Severe COVID-19: A Retrospective Observational Study. Radiology 2020;202222

20 Asadi-Pooya AA, Simani L. Central nervous system manifestations of COVID-19: A systematic review. J Neurol Sci 2020;413:116832

21 Autopsy findings and venous thromboembolism in patients with COVID-19: a prospective cohort study. Available at: https://www.acpjournals.org/doi/full/10.7326/M20-2003. Accessed July 31, 2020 
22 Acute ischemic stroke and COVID-19. Available at: https:// www.ccjm.org/content/early/2020/06/01/ccjm.87a.ccc042. Accessed July 31, 2020

23 Zhang Y, Xiao M, Zhang S, et al. Coagulopathy and antiphospholipid antibodies in patients with Covid-19. N Engl J Med 2020;382(17):e38

24 AlKetbi R, AlNuaimi D, AlMulla M, et al. Acute myelitis as a neurological complication of Covid-19: a case report and MRI findings. Radiol Case Rep 2020;15(9):1591-1595

25 Patel ZM, Fernandez-Miranda J, Hwang PH, et al. Letter: precautions for endoscopic transnasal skull base surgery during the COVID-19 pandemic. Neurosurgery 2020;87(1):E66-E67

26 Özdin S, Bayrak Özdin Ş. Levels and predictors of anxiety, depression and health anxiety during COVID-19 pandemic in Turkish society: The importance of gender. Int J Soc Psychiatry 2020;66(5):504-511

27 Chew NWS, Lee GKH, Tan BYQ et al. A multinational, multicentre study on the psychological outcomes and associated physical symptoms amongst healthcare workers during COVID-19 outbreak. Brain Behav Immun 2020;88:559-565

28 La Vignera S, Cannarella R, Condorelli RA, Torre F, Aversa A, Calogero AE. Sex-specific SARS-CoV-2 mortality: among hormone-modulated ACE2 expression, risk of venous thromboembolism and hypovitaminosis D. Int J Mol Sci 2020;21(8):E2948

29 Dalpiaz PLM, Lamas AZ, Caliman IF, et al. Sex hormones promote opposite effects on ACE and ACE2 activity, hypertrophy and cardiac contractility in spontaneously hypertensive rats. PLoS One 2015;10(5):e0127515

30 Sex hormones and novel Corona virus infectious disease (COVID-19). Available at: https://reader.elsevier. com/reader/sd/pii/S0025619620305188?token $=$ CF6B93EE45 666910ADEDDFA5E815225999B1116EFD86CE6C9D0324 E72A00C500E073037031E00254B8182D66E7B60A1C. Accessed July 31, 2020

31 Traish A, Bolanos J, Nair S, Saad F, Morgentaler A. Do androgens modulate the pathophysiological pathways of inflammation? Appraising the contemporary evidence. J Clin Med 2018;7(12):E549

32 Brownlee W, Bourdette D, Broadley S, Killestein J, Ciccarelli O. Treating multiple sclerosis and neuromyelitis optica spectrum disorder during the COVID-19 pandemic. Neurology 2020;94(22):949-952

33 Cilia R, Bonvegna S, Straccia G, et al. Effects of COVID-19 on Parkinson's disease clinical features: a community-based case-control study. Mov Disord 2020. (e-pub ahead of print). doi: https://doi.org/10.1002/mds.28170

34 Psychiatric manifestations of neurologic disease: where are we headed? Available at: https://www.ncbi.nlm.nih.gov/pmc/ articles/PMC2687521/. Accessed July 31, 2020

35 Bhatia R, Sylaja PN, Srivastava MP, et al. Consensus statement - suggested recommendations for acute stroke management during the COVID-19 pandemic: Expert group on behalf of the Indian stroke association. Ann Indian Acad Neurol 2020;23(7):15-23

36 Bhatia R, Srivastava MVP, Khurana D, et al. Consensus statement on immune modulation in multiple sclerosis and related disorders during the COVID-19 pandemic: Expert Group on Behalf of the Indian Academy of Neurology. Ann Indian Acad Neurol 2020;23(Suppl 1) :S5-S14

37 Juurlink DN. Safety considerations with chloroquine, hydroxychloroquine and azithromycin in the management of SARSCoV-2 infection. CMAJ 2020;192(17):E450-E453

38 Ong JJ, Bharatendu C, Goh Y, et al Headaches associated with personal protective equipment - A cross - sectional study among frontline healthcare workers during COVID - 19. Headache J Head Face Pain. 2020;60(5):864864

39 Szczesniak D, Ciulkowicz M, Maciaszek J, et al. Psychopathological responses and face mask restrictions during the COVID-19 outbreak: results from a nationwide survey. Brain Behav Immun 2020;87:161-162 